\title{
Whole-body magnetic resonance imaging for the evaluation of thoracic involvement in disseminated paracoccidioidomycosis
}

\author{
Ressonância magnética de corpo inteiro na avaliação do \\ comprometimento torácico na paracoccidioidomicose disseminada
}

\author{
Edson Marchiori, Elisa Carvalho Ferreira, Gláucia Zanetti, Bruno Hochhegger
}

\section{To the Editor:}

Whole-body magnetic resonance imaging (MRI) is a diagnostic tool that enables the visualization of the entire body and combines fast acquisition times with high contrast resolution. An important advantage of this technique is the absence of ionizing radiation. Thus, whole-body MRI offers a radiation-free alternative to radionuclide imaging and $\mathrm{CT}$ for the diagnosis, staging, and monitoring of young patients who require frequent follow-up examinations. Because MRI is the best method to evaluate bone marrow and soft tissues, it represents an ideal tool for the assessment of numerous systemic diseases, especially those affecting the skeletal system..$^{(1)}$

Paracoccidioidomycosis, a systemic fungal disease caused by the fungus Paracoccidioides brasiliensis, is endemic in Central and South America. ${ }^{(2,3)}$ In Europe and North America, it is considered a rare imported disease, the incidence of which is expected to continue rising due to the increase in migration and international travel. ${ }^{(4)}$ Given that the disease can affect virtually any organ, whole-body MRl seems to be a suitable diagnostic tool for evaluating systemic manifestations of disseminated paracoccidioidomycosis, because it provides a global view. The aim of the present report was to present the whole-body MRI findings of a patient with systemic manifestations of paracoccidioidomycosis, with an emphasis on lymphatic, skeletal, and pleural involvement. To our knowledge, thoracic findings of disseminated paracoccidioidomycosis on whole-body MRI have not been described previously.

A 24-year-old man was referred to our facility for the diagnostic investigation of multiple complaints. The symptoms began approximately 15 months prior to hospitalization and mainly consisted of emaciation, fever, painful lymphadenopathy, and crusted, ulcerated skin lesions. The epidemiologic history of the patient revealed that he had worked for the past two years as a farm worker, taking care of animals.

Upon physical examination, the patient presented with fever, multiple honey-colored scabs, and ulcerated lesions on the face, trunk, upper limbs, and perianal region. Pulmonary auscultation findings were normal, as were those of the cardiac examination, and there was no abdominal organomegaly.

A left cervical lymph node was aspirated, and direct examination for fungi revealed refringent blastoconidia with multiple buds suggestive of $P$. brasiliensis in the specimen. Blood agar culture was also positive for $P$. brasiliensis. A skin biopsy specimen stained with Grocott and periodic acidSchiff stains revealed yeast cells with multiple buds in the form of a ship's wheel, which is a typical finding of $P$. brasiliensis. Serology by double immunodiffusion techniques was positive for paracoccidioidomycosis. A blood test for HIV was negative. A diagnosis of the acute or subacute so-called "juvenile" type of disseminated paracoccidioidomycosis was made, and the patient was hospitalized for treatment.

Before the necessary treatment was started, written informed consent was obtained and the patient was submitted to whole-body MRI in order to further investigate the systemic involvement of the disseminated disease. The examination revealed extensive involvement at multiple locations throughout the body, including the subcutaneous tissue, lymph nodes, skeletal system, pleural cavity, and peritoneal cavity. Lymph node enlargement was seen on whole-body MRI in multiple lymph node chains. On a gadoliniumenhanced volumetric interpolated breath-hold examination (VIBE) sequence, the lymph nodes presented peripheral contrast enhancement (Figure $1 \mathrm{~A})$. On a short-tau inversion-recovery (STIR) sequence, the central portions of those 
lymph nodes exhibited hyperintense signals, giving them a necrotic appearance.

On T1-weighted images, the skeletal lesions varied from hypointense to isointense, exhibiting, at some sites, cortical bone destruction and an extensive soft tissue component. In a gadoliniumenhanced VIBE sequence with fat suppression, the lesions exhibited an insufflating pattern, with contrast enhancement predominantly in the peripheral regions (Figures $1 \mathrm{~B}$ and $2 \mathrm{~A}$ ). On a STIR sequence, the central portions of the lesions presented hyperintense signals, suggestive of necrotic tissue.

Whole-body MRI with STIR sequences also revealed pleural and peritoneal involvement, manifesting as pleural effusion and ascites, as evidenced by a typical hyperintense signal (Figure 2B). There were no other sites of involvement.

Paracoccidioidomycosis is divided into two polar forms, depending on its epidemiological, immunopathological, and clinical aspects. ${ }^{(5)}$ The acute or subacute form most frequently affects children, teenagers, and young adults and is known as the juvenile type. It manifests as extensive involvement of the mononuclear phagocytic system, mainly in the spleen, liver, lymph nodes, and intestinal tract. Lung and mucosal lesions are rare. ${ }^{(5)}$ The chronic form, known as the adult type, is more common, accounting for 90\% of all cases, and predominantly affects adults over 30 years of age. The chronic form is more likely to be localized than is the acute form. Initially, the disease is localized in the lungs, and less frequently, in the mucosa or skin..$^{(5)}$ Therefore, our case can be classified as the acute form of paracoccidioidomycosis. ${ }^{(3)}$

In the case presented here, whole-body MRl showed involvement at multiple locations throughout the body, including the subcutaneous tissue, lymph nodes, skeletal system, pleural cavity, and peritoneal cavity. Of the systemic manifestations, we focused on the extensive thoracic skeletal involvement. Bone and joint infections are relatively uncommon in patients with paracoccidioidomycosis. The lesions are described as circumscribed, well-delineated areas of lysis, with or without a rim of sclerosis that can occur in any bone, although such lesions are usually seen in clavicles, ribs, and scapulae. ${ }^{(6)}$

Although the patient had no symptoms of skeletal involvement, we decided to perform whole-body MRI in order to conduct a detailed investigation. Whole-body MRI seemed suitable for the case presented here, because, in addition to its high contrast resolution and excellent ability to visualize bone marrow components, the method has the advantage of not using ionizing radiation, making it an ideal tool for the initial evaluation and follow-up assessment of children and young adults such as our patient. ${ }^{(7)}$

In conclusion, the case presented here illustrates the potential of whole-body MRI as a diagnostic tool for evaluating systemic diseases, such as deep mycosis. This radiation-free evaluative method creates images that can be used as baseline assessments for longitudinal monitoring.

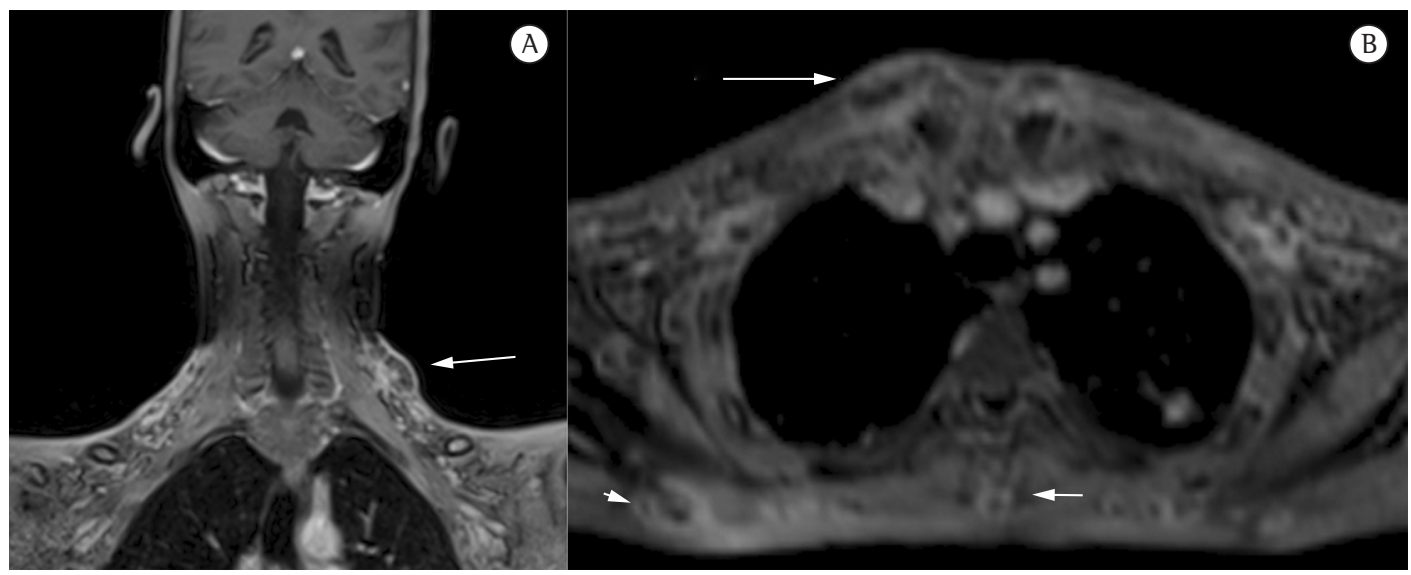

Figure $\mathbf{1}$ - Whole-body magnetic resonance imaging using a gadolinium-enhanced volumetric interpolated breath-hold examination sequence. In A, a scan demonstrating the enlargement of a cervical lymph node and peripheral contrast enhancement (arrows). In B, a scan in the axial plane showing insufflated lesions on the clavicle (long arrow), scapula (arrowhead), and vertebra (short arrow). 


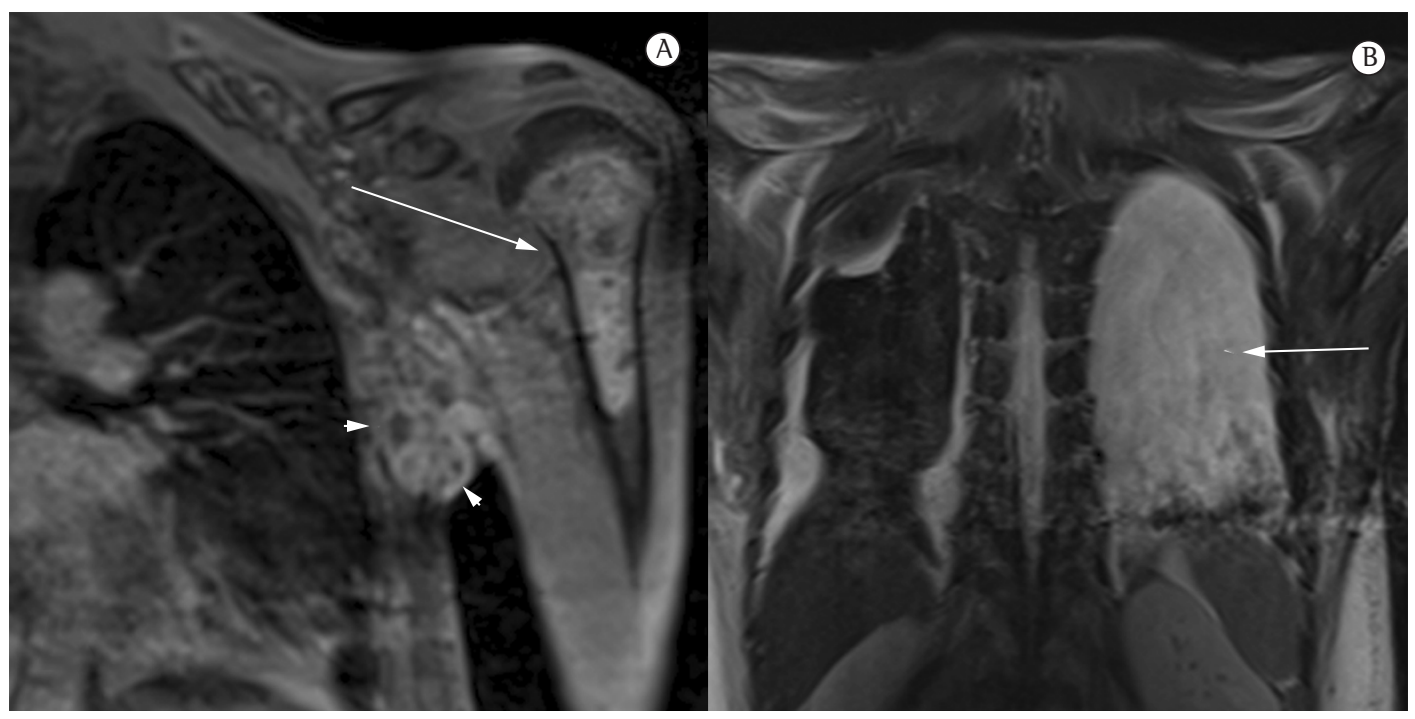

Figure 2 - Whole-body magnetic resonance imaging scans in the coronal plane. In A, lesions in the left humerus (arrow) with heterogeneous contrast enhancement, and axillary lymph node enlargement (arrowheads). In B, bilateral pleural effusion (arrow).

Edson Marchiori

Associate Professor of Radiology, Federal University of Rio de Janeiro, Rio de Janeiro, Brazil

Elisa Carvalho Ferreira

Radiologist, Hospital de Ipanema, Rio de Janeiro, Brazil

\section{Gláucia Zanetti}

Professor of Clinical Medicine, Petrópolis School of Medicine, Petrópolis, Brazil

\section{Bruno Hochhegger}

Chest Radiologist, Santa Casa Hospital Complex, Porto Alegre, Brazil

\section{References}

1. Ferreira EC, Brito CC, Domingues RC, Bernardes M, Marchiori E, Gasparetto EL. Whole-body MR imaging for the evaluation of McCune-albright syndrome. J Magn Reson lmaging. 2010;31(3):706-10.

2. Martinez R. Paracoccidioidomycosis: the dimension of the problem of a neglected disease. Rev Soc Bras Med Trop. 2010;43(4):480.

3. Nogueira SA, Guedes AL, Wanke B, Capella S, Rodrigues K, Abreu TF, et al. Osteomyelitis caused by Paracoccidioides brasiliensis in a child from the metropolitan area of Rio de Janeiro. J Trop Pediatr. 2001;47(5):311-5.

4. Buitrago MJ, Bernal-Martínez L, Castelli MV, RodríguezTudela JL, Cuenca-Estrella M. Histoplasmosis and paracoccidioidomycosis in a non-endemic area: a review of cases and diagnosis. J Travel Med. 2011;18(1):26-33.

5. Valera ET, Mori BM, Engel EE, Costa 1S, Brandão DF, Nogueira-Barbosa $\mathrm{MH}$, et al. Fungal infection by Paracoccidioides brasiliensis mimicking bone tumor. Pediatr Blood Cancer. 2008;50(6):1284-6.

6. Nogueira MG, Andrade GM, Tonelli E. Clinical evolution of paracoccidioidomycosis in 38 children and teenagers. Mycopathologia. 2006;161(2):73-81.

7. Ley S, Ley-Zaporozhan J, Schenk JP. Whole-body MRI in the pediatric patient. Eur J Radiol. 2009;70(3):442-51. 